\title{
HD 146875: a newly identified ZAMS active double-lined spectroscopic binary (Research Note)
}

\author{
H. J. Wang ${ }^{1,2}$, J. Y. Wei ${ }^{1}$, J. R. Shi ${ }^{1}$, and J. K. Zhao ${ }^{1}$ \\ 1 National Astronomical Observatories, Chinese Academy of Sciences, Beijing 100012, PR China \\ e-mail: wanghj@bao.ac.cn \\ ${ }^{2}$ Graduate University of the Chinese Academy of Sciences, Beijing 100049, PR China
}

Received 28 August 2008 / Accepted 10 April 2009

\section{ABSTRACT}

\begin{abstract}
Aims. We present a newly identified zero-age main-sequence (ZAMS) active double-lined spectroscopic binary, HD 146875, based on the analysis of our 45 high-resolution spectra obtained between 2005 and 2008 at Xinglong Observatory, NAOC.

Methods. From spectroscopic analysis and abundance analysis, we derived the accurate orbital parameters of the binary and determined the stellar atmospheric parameters of the two components.

Results. Our results indicate that HD 146875 is a ZAMS active double-lined spectroscopic binary of age between 100 and 150 Myr. We derived the accurate orbital parameters of the binary and determined the stellar atmospheric parameters of the two components. The photometric observations taken in 2008 show a variation of about 0.057 mag caused by large spots most probably on the primary component of the binary rather than any ellipsoidal distortion. Furthermore, the radial velocity curve and the light curve imply that HD 146875 is already a synchronous system. We also studied the chromospheric activity of the components.
\end{abstract}

Key words. stars: activity - stars: binaries: spectroscopic - stars: starspots - stars: late-type - stars: individual: HD 146875

\section{Introduction}

Spectroscopic observations of binaries provide the opportunity to derive precise measurements of the fundamental stellar parameters of their constituent stars such as stellar masses and radii. Although there have been advances in determining the masses of young low-mass $\left(M \leq 2 M_{\odot}\right)$ stars, the sample of well-studied young low-mass binaries remains small. An expansion of this sample is therefore needed to derive more robust calibrations of young-star evolutionary models, and to determine the unbiased mass-ratio and orbital-period distribution of young low-mass double-lined spectroscopic binaries (SB2s) (Prato et al. 2002). High-resolution spectroscopic observation is a powerful technique for discovering binaries and providing accurate parameters, especially for masses and mass ratio of the components.

HD 146875 is a bright X-ray source selected from the ROSAT bright source catalogue (Schwope et al. 2000). According to SIMBAD, its $V$-band magnitude is $8.81 \mathrm{mag}$. However no high-resolution spectroscopic observation has been presented before, and this is the first time that it has been identified as a SB2.

Our attention was drawn toward HD 146875 because of the clear close double-lines in the high-resolution spectrum obtained on April 25, 2005 to identify pre-main-sequence (PMS) stars, and we reobserved the star on February 17, 2006 and June 9, 2006. All these high-resolution spectra confirm that it is a double-lined spectroscopic binary. Furthermore, its strong X-ray and Ca II infrared triplet (IRT) line emission and strong Li I absorption increased our interest in this binary. To compile its radial velocity (RV) curve and more accurate parameters, the spectroscopic observations continued until March 25, 2008. We also performed photometric observations.
We present the details of our observations and data reduction in Sect. 2, the analysis of the observational data in Sect. 3, and our conclusions in Sect. 4.

\section{Observations and data reduction}

\subsection{Spectroscopic observations}

High-resolution spectroscopic observations of HD 146875 were performed 45 times during 13 nights in the past 4 years (see Col. 1 of Table 1) using the Coude echelle spectrograph mounted on the $2.16 \mathrm{~m}$ telescope at Xinglong Observatory of National Astronomical Observatories, Chinese Academy of Science (NAOC). The detector is a TEK CCD with $1024 \times$ 1024 pixels of $24 \times 24 \mu \mathrm{m}^{2}$. About 41 spectral orders are included in these spectra that range in wavelength from $5850 \AA$ to $9450 \AA$, with a normal resolving power of $\lambda / \Delta \lambda \simeq 40000$. Technical details about this Coude echelle spectrograph can be found in Zhao et al. (2001). The exposure time of each spectrum being one hour and the signal-to-noise ratio of the acquired data is above 100 about $\mathrm{H} \alpha$ line for most of these spectra (none below 80). At least two RV standard stars were observed each night, except for February 17, 2006 on which only one RV standard star was observed.

The spectra were reduced using the ECHELLE package in $\mathrm{IRAF}^{1}$, flat-fielded using dome flats, and wavelength-calibrated using Th-Ar lamp spectra. The precision of the wavelength calibration is about $0.003 \AA$. Finally, the spectra were normalized by a polynomial fit.

\footnotetext{
${ }^{1}$ IRAF is distributed by the National Optical Astronomy Observatory, which is operated by the Association of Universities for Research in Astronomy, Inc., under cooperative agreement with the National Science Foundation.
} 
Table 1. Radial velocities of the components.

\begin{tabular}{|c|c|c|c|c|}
\hline Obs. Date & $\begin{array}{c}\text { HJD } \\
2450000+\end{array}$ & $\begin{array}{c}\mathrm{RV}_{\mathrm{p}} \\
\mathrm{km} \mathrm{s}^{-1}\end{array}$ & $\begin{array}{c}\mathrm{RV}_{\mathrm{s}} \\
\mathrm{km} \mathrm{s}^{-1}\end{array}$ & $\overline{\overline{\text { Phase }^{a}}}$ \\
\hline 2005.04 .25 & 3486.18864 & -52.8 & -23.7 & 0.586 \\
\hline \multirow[t]{2}{*}{2006.02 .17} & 3784.34546 & -31.3 & -48.9 & 0.171 \\
\hline & 3784.38736 & -31.9 & -48.0 & 0.176 \\
\hline \multirow[t]{2}{*}{ 2006.06.09 } & 3896.13871 & -28.9 & -52.4 & 0.139 \\
\hline & 3896.18125 & -29.2 & -51.9 & 0.144 \\
\hline \multirow[t]{4}{*}{ 2007.04.24 } & 4215.14663 & -29.7 & -52.2 & 0.143 \\
\hline & 4215.18909 & -29.9 & -51.8 & 0.148 \\
\hline & 4215.23523 & -30.2 & -51.1 & 0.153 \\
\hline & 4215.27864 & -30.5 & -50.7 & 0.158 \\
\hline \multirow[t]{8}{*}{2007.04 .25} & 4216.11403 & -39.6 & -39.6 & 0.255 \\
\hline & 4216.15682 & -39.8 & -39.8 & 0.260 \\
\hline & 4216.17856 & -39.9 & -39.9 & 0.262 \\
\hline & 4216.20054 & -40.0 & -40.0 & 0.265 \\
\hline & 4216.22228 & -40.1 & -40.1 & 0.268 \\
\hline & 4216.24334 & -40.2 & -40.2 & 0.270 \\
\hline & 4216.26712 & -40.4 & -40.4 & 0.273 \\
\hline & 4216.30915 & -40.6 & -40.6 & 0.278 \\
\hline \multirow[t]{4}{*}{ 2007.04.26 } & 4217.11808 & -50.2 & -25.6 & 0.371 \\
\hline & 4217.22804 & -51.0 & -24.7 & 0.384 \\
\hline & 4217.27039 & -51.2 & -24.2 & 0.389 \\
\hline & 4217.31297 & -51.4 & -23.6 & 0.394 \\
\hline \multirow[t]{3}{*}{ 2007.04.27 } & 4218.19149 & -55.8 & -21.6 & 0.496 \\
\hline & 4218.27938 & -55.3 & -20.9 & 0.506 \\
\hline & 4218.31466 & -55.3 & -21.2 & 0.510 \\
\hline \multirow[t]{6}{*}{2007.05 .01} & 4222.08975 & -24.0 & -57.3 & 0.948 \\
\hline & 4222.13194 & -23.9 & -57.4 & 0.953 \\
\hline & 4222.17564 & -23.7 & -57.3 & 0.958 \\
\hline & 4222.21759 & -23.7 & -57.4 & 0.963 \\
\hline & 4222.25955 & -23.5 & -57.4 & 0.968 \\
\hline & 4222.30160 & -23.4 & -57.4 & 0.973 \\
\hline \multirow[t]{5}{*}{2007.05 .03} & 4224.09796 & -32.7 & -49.1 & 0.181 \\
\hline & 4224.14012 & -33.3 & -49.0 & 0.186 \\
\hline & 4224.18199 & -33.9 & -48.5 & 0.191 \\
\hline & 4224.22404 & -34.0 & -47.1 & 0.196 \\
\hline & 4224.26593 & -35.0 & -46.0 & 0.201 \\
\hline \multirow[t]{3}{*}{2008.02 .16} & 4513.31773 & -40.8 & -40.8 & 0.730 \\
\hline & 4513.36034 & -40.5 & -40.5 & 0.735 \\
\hline & 4513.40304 & -40.3 & -40.3 & 0.740 \\
\hline \multirow[t]{2}{*}{2008.02 .17} & 4514.34796 & -30.3 & -51.3 & 0.849 \\
\hline & 4514.39059 & -29.9 & -51.8 & 0.854 \\
\hline \multirow[t]{2}{*}{2008.02 .18} & 4515.33964 & -23.8 & -57.1 & 0.964 \\
\hline & 4515.38175 & -23.7 & -57.3 & 0.969 \\
\hline \multirow{2}{*}{2008.03 .25} & 4551.21498 & -28.3 & -53.4 & 0.126 \\
\hline & 4551.25745 & -28.7 & -52.7 & 0.131 \\
\hline
\end{tabular}

${ }^{a}$ The orbital phase based on a period of 8.6209 days corresponding to HJD $=2454213.9161$.

\subsection{Photometric observations}

Photometric observations of HD 146875 were performed from February 15, 2008 to April 13, 2008 on the $60 \mathrm{~cm}$ reflecting telescope at Xinglong Observatory of NAOC. The detector is a E2V CCD with $1024 \times 1024$ pixels of $13.5 \times 13.5 \mu \mathrm{m}^{2}$. The field of view is $17^{\prime} \times 17^{\prime}$ and standard Johnson $\mathrm{V}$ filter was used. A total of $6280 \mathrm{~V}$-band measurements were obtained during 21 nights with a typical photometric precision of $0.01 \mathrm{mag}$.

The photometric data were reduced using the APPHOT package in IRAF. In total 5685 good data points were obtained after removing bad data points caused by poor weather. In order to increase the signal-to-noise ratio of the light-curve data, we combined every 5 data points, finally obtaining 1137 points for our data analysis. The photometric period was obtained using the PDM task in IRAF, based on the method of the phase dispersion minimization (Stellingwerf 1978).

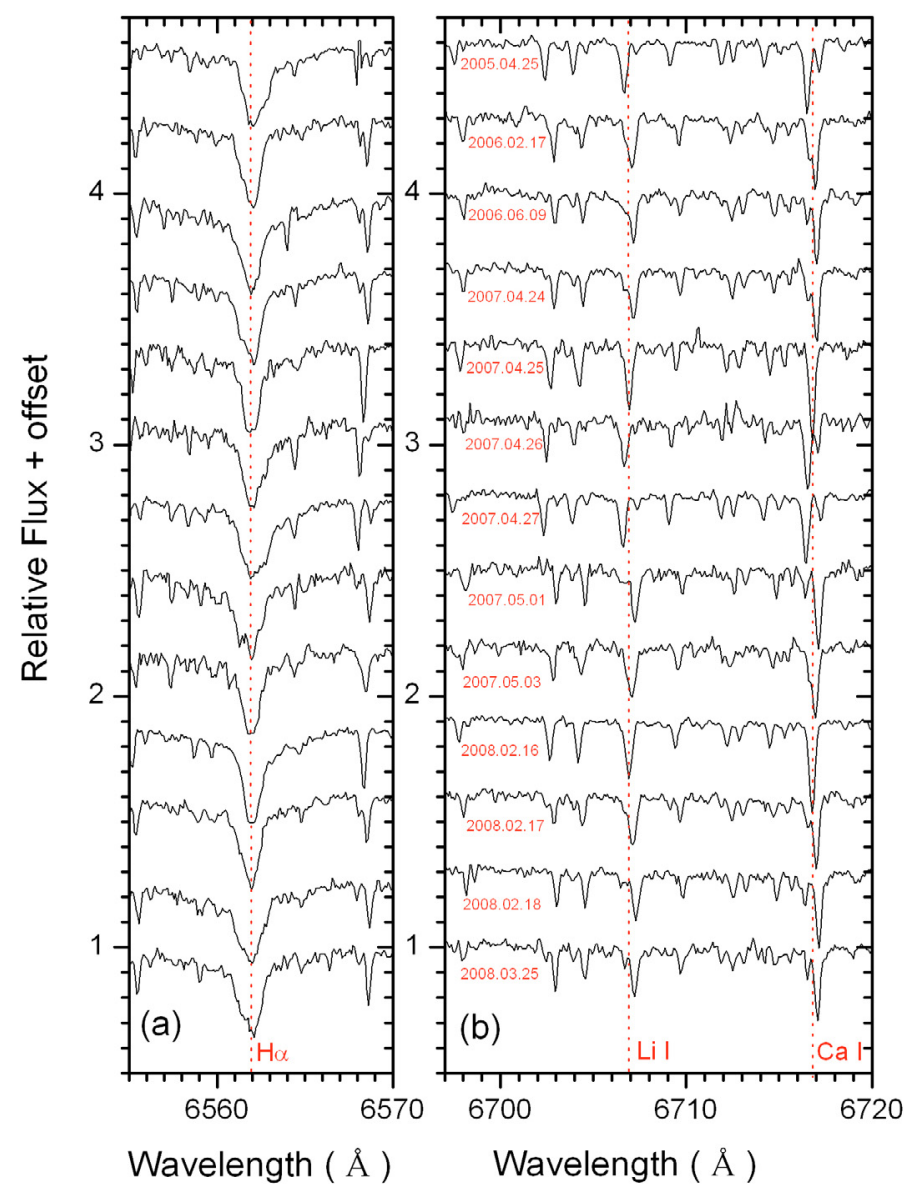

Fig. 1. High-resolution optical spectra of HD 146875 in the regions of a) $\mathrm{H} \alpha(6563 \AA)$ and b) Li I $(6708 \AA)$ lines. All the spectra have been adjusted to heliocentric velocities. The H $\alpha$, Li I, and Ca I (6718 ̊) absorption lines are indicated by dot lines and the observational date of each spectrum is also marked.

\section{Analysis of the observational data}

\subsection{Direct identification from the high-resolution spectra}

H $\alpha(6563 \AA)$ and Li I (6708 $\AA$ ) lines of some spectra are presented in Fig. 1, and Ca II IRT lines in Fig. 2. All the spectra were corrected to a heliocentric frame of reference. The centroid of the binary for these lines is indicated in both figures by dotted lines and the observational date of each spectrum is also marked. We were able to infer the relative movement between the double lines directly from the spectra in Fig. 1, especially around the strong and narrow lines, such as Ca I (6718 $\AA$ ), and therefore that it is a double-lined spectroscopic binary. Because of its strong X-ray emission and obvious Ca II IRT emission, it is an active spectroscopic binary. On the basis of its Li I absorption, the star appears to be young.

\subsection{Spectral disentangling}

The spectral disentangling technique was adopted to extract the individual contribution of the two components. We used the technique developed by Hadrava (1995) and applied within the KOREL code (from personal communication). We show the separated spectra of the components in the regions of $\mathrm{H} \alpha, \mathrm{Li}$ I, and Ca II IRT in Figs. 3, 4, and 5, respectively. 


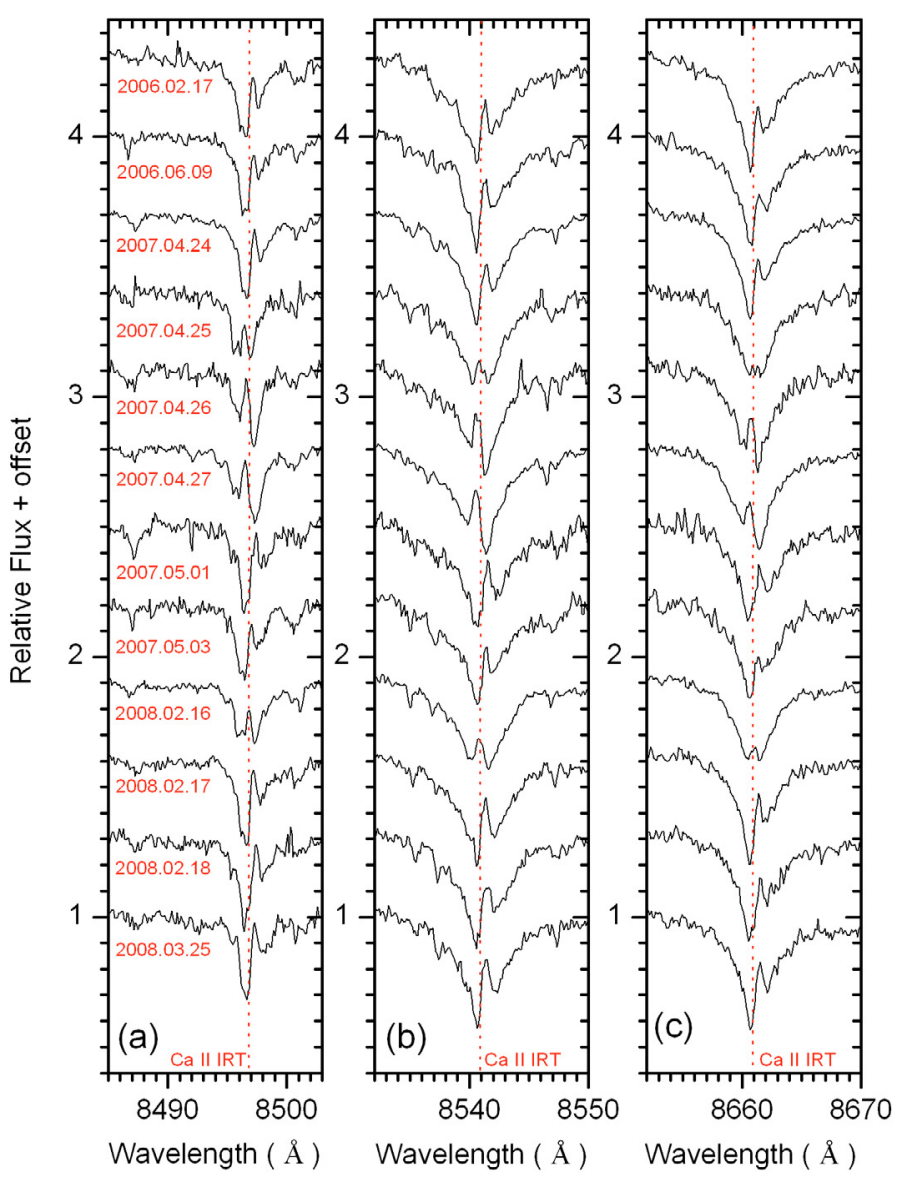

Fig. 2. High-resolution optical spectra of HD 146875 in the regions of Ca II IRT (a) $8498 \AA$ b) $8542 \AA$ c) $8662 \AA$ ) lines. All the spectra have been adjusted to heliocentric velocities. These three lines are marked with dot lines and the observational date of each spectrum is also marked.

\subsection{Orbital parameters derived by KOREL}

We list the orbital parameters derived by KOREL in Table 2. The orbital parameters are the period, $P$, the time of the primary receding at maximum radial velocity, $T_{0}$, the eccentricity, $e$, the semi-amplitudes of the radial velocity, $K_{\mathrm{P}}$ and $K_{\mathrm{S}}$, the mass ratio, $q$, and the total uncertainty in the analysis of these parameters, rms. The quantities derived based on these parameters are also listed in Table 2, including the projected semi-major axes, $a \sin i$, and the minimum masses of the components, $M \sin ^{3} i$.

\subsection{Stellar atmospheric parameters}

To derive the stellar atmospheric parameters, we measured the equivalent widths (EWs) of $46 \mathrm{Fe} \mathrm{I}$ and $6 \mathrm{Fe}$ II lines of the primary and $47 \mathrm{Fe}$ I and $5 \mathrm{Fe}$ II lines of the secondary from well separated spectra. The uncertainty in our EW measurement was about 10 percent for the primary component and 20 percent for the secondary component, which originated mostly from the identification of the spectrum continuum. All the EW were then corrected for the difference in continuum fluxes between the two components (based on the ratio of the peak intensities in the cross-correlation function between the binary spectrum and the spectrum of a standard star of similar spectral type) (Cargile et al. 2008; Covino et al. 2001).

The stellar atmospheric parameters including the effective temperature, $T_{\text {eff }}$, the metallicity, $[\mathrm{Fe} / \mathrm{H}]$, the gravity, $\log g$, and

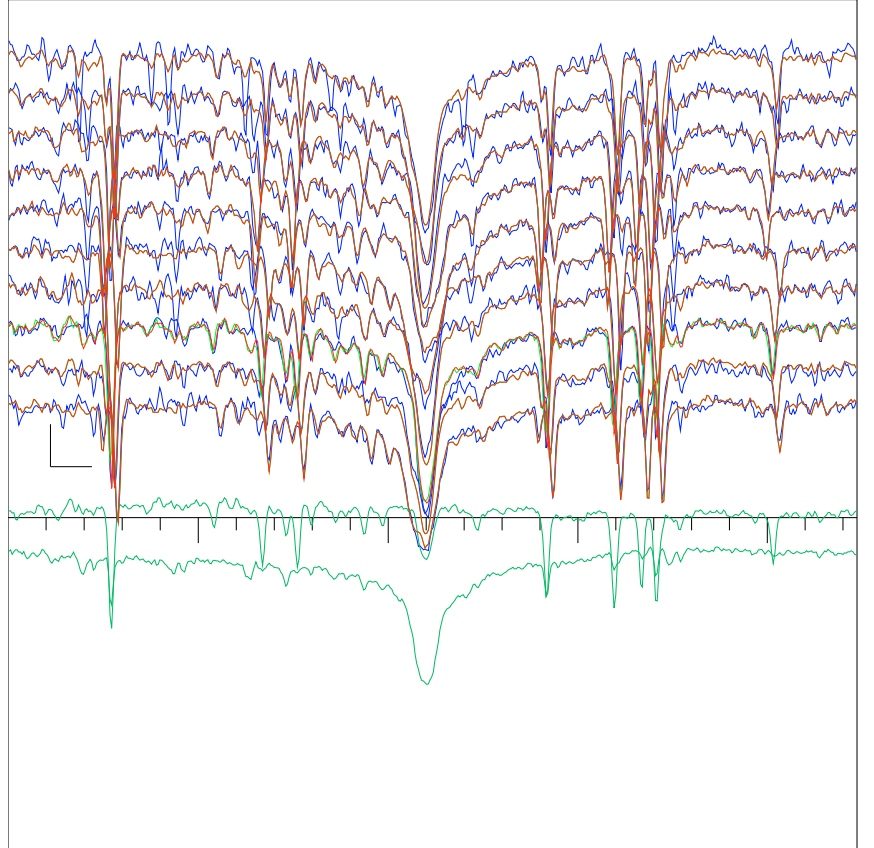

Fig. 3. Separated $\mathrm{H} \alpha$ lines of the components. The upper 10 lines correspond to the input spectra ordered in running time from the top. These profiles are superimposed on their reconstruction as the sum of the separated components. The L-shaped line in the figure indicates the wavelength unit $\left(100 \mathrm{~km} \mathrm{~s}^{-1}\right)$ and the unit of intensity $(0.1$ of the continuum level).

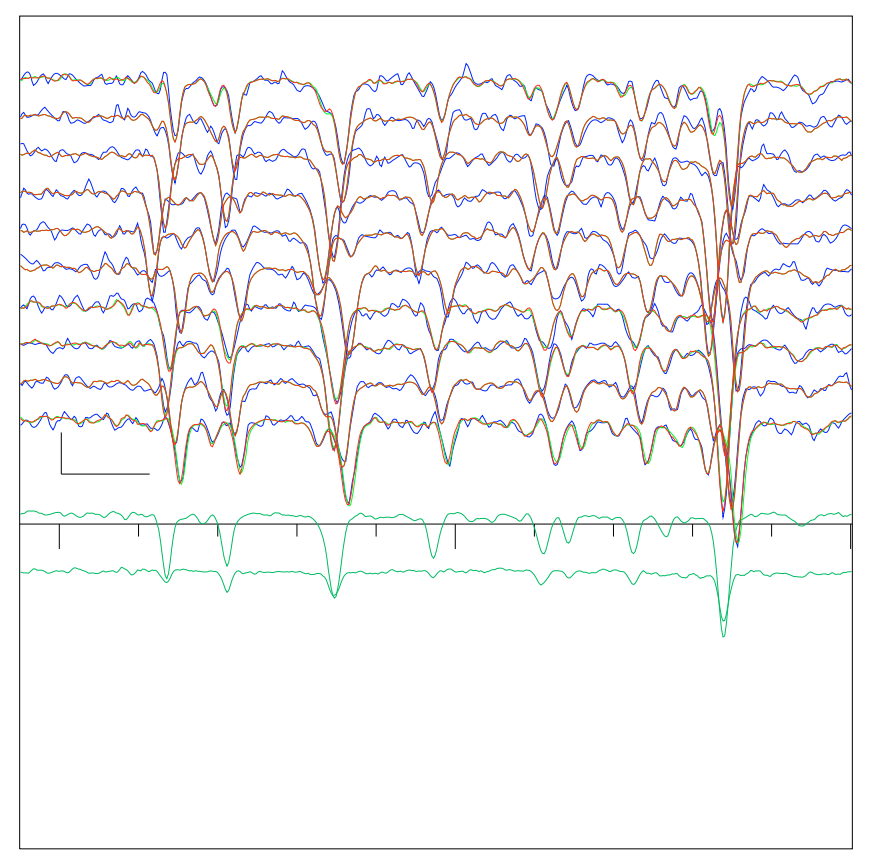

Fig. 4. The same as Fig. 3 but in the region of Li I.

the microturbulence, $\xi$ were derived using the routine for abundance analysis developed by Zhao et al. (2007). In this routine, the effective temperature is determined from an abundance derived from Fe I lines with different excitation potentials. The errors in the temperature are mostly induced by incorrect damping parameters (Ryan 1998) or non-LTE effects, and can be as high as $100 \mathrm{~K}$ (Chen 2000). Because of the 10 percent uncertainty in the EW measurements of the primary and 20 percent for the secondary, the error in temperature could reach $150 \mathrm{~K}$ for the 


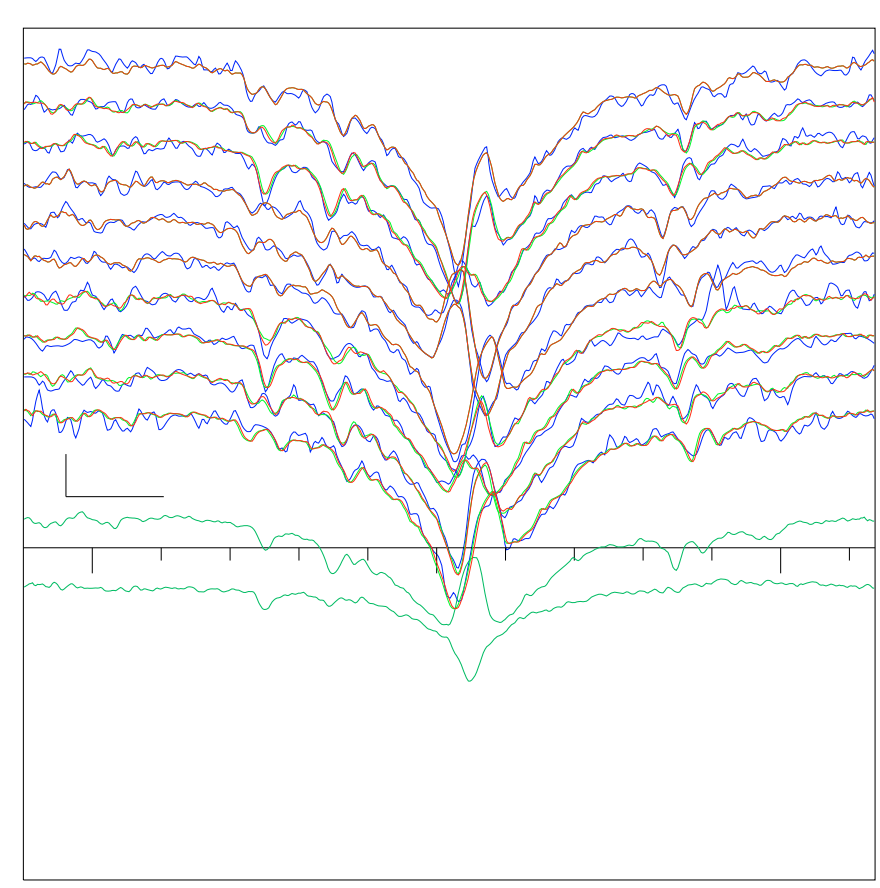

Fig. 5. The same as Fig. 3 but in the region of CaII IRT (8542 A).

Table 2. Parameters derived based on KOREL.

\begin{tabular}{|c|c|}
\hline Parameter & $\overline{\text { Value }}$ \\
\hline$P$ (days) & $8.6230 \pm 0.0001$ \\
\hline$T_{0}^{a}$ & $2454213.9161 \pm 0.0001$ \\
\hline$e$ & $0.000 \pm 0.001$ \\
\hline$K_{\mathrm{P}}\left(\mathrm{km} \mathrm{s}^{-1}\right)$ & $16.3 \pm 0.2$ \\
\hline$K_{\mathrm{S}}\left(\mathrm{km} \mathrm{s}^{-1}\right)$ & $19.0 \pm 0.7$ \\
\hline$q$ & $0.855 \pm 0.001$ \\
\hline $\mathrm{rms}$ & 0.197 \\
\hline$a \sin i\left(R_{\odot}\right)$ & $6.01 \pm 0.02$ \\
\hline$M_{\mathrm{P}} \sin ^{3} i\left(M_{\odot}\right)$ & $0.0283 \pm 0.0002$ \\
\hline$M_{\mathrm{S}} \sin ^{3} i\left(M_{\odot}\right)$ & $0.0242 \pm 0.0002$ \\
\hline$T_{\text {eff }_{\mathrm{P}}}(\mathrm{K})$ & $5185 \pm 150$ \\
\hline$T_{\text {eff }}(\mathrm{K})$ & $4930 \pm 200$ \\
\hline $\log g_{\mathrm{P}}$ & $4.4 \pm 0.1$ \\
\hline $\log g_{\mathrm{S}}$ & $4.5 \pm 0.4$ \\
\hline$[\mathrm{Fe} / \mathrm{H}]_{\mathrm{P}}$ & $0.0 \pm 0.1$ \\
\hline$[\mathrm{Fe} / \mathrm{H}]_{\mathrm{S}}$ & $-1.0 \pm 0.3$ \\
\hline$\xi_{\mathrm{P}}\left(\mathrm{km} \mathrm{s}^{-1}\right)$ & $1.3 \pm 0.3$ \\
\hline$\xi_{\mathrm{s}}\left(\mathrm{km} \mathrm{s}^{-1}\right)$ & $0.9 \pm 0.6$ \\
\hline$E W(\mathrm{Li})_{\mathrm{P}}(\mathrm{m} \AA)$ & $135 \pm 13$ \\
\hline$E W(\mathrm{Li})_{\mathrm{S}}(\mathrm{m} \AA)$ & $52 \pm 10$ \\
\hline $\log N(\mathrm{Li})_{\mathrm{P}}$ & $2.5 \pm 0.1$ \\
\hline $\log N(\mathrm{Li})_{\mathrm{S}}$ & $1.5 \pm 0.3$ \\
\hline$M_{\text {bolp }}(\mathrm{mag})$ & $5.4 \pm 0.5$ \\
\hline$M_{\text {bols }}(\mathrm{mag})$ & $6.1 \pm 1.0$ \\
\hline$M_{\mathrm{V}_{\mathrm{P}}}(\mathrm{mag})$ & $5.8 \pm 0.5$ \\
\hline$M_{\mathrm{V}_{\mathrm{S}}}(\mathrm{mag})$ & $6.4 \pm 1.0$ \\
\hline$M_{\mathrm{P}}\left(M_{\odot}\right)$ & $0.8 \pm 0.1$ \\
\hline$M_{\mathrm{S}}\left(M_{\odot}\right)$ & $0.7 \pm 0.2$ \\
\hline$R_{\mathrm{P}}\left(R_{\odot}\right)$ & $0.9 \pm 0.2$ \\
\hline$R_{\mathrm{S}}\left(R_{\odot}\right)$ & $0.8 \pm 0.4$ \\
\hline$i$ (degree) & $19.1 \pm 0.8$ \\
\hline$D(\mathrm{PC})$ & $51 \pm 13$ \\
\hline$S p T_{\mathrm{P}}$ & $\mathrm{K} 0 \pm 1$ subclass \\
\hline$S p T_{\mathrm{S}}$ & $\mathrm{K} 2 \pm 1$ subclass \\
\hline$V_{\mathrm{P}} \sin i\left(\mathrm{~km} \mathrm{~s}^{-1}\right)$ & $5 \pm 1$ \\
\hline$V_{\mathrm{S}} \sin i\left(\mathrm{~km} \mathrm{~s}^{-1}\right)$ & $4 \pm 2$ \\
\hline
\end{tabular}

${ }^{a}$ The time of maximum primary velocity.

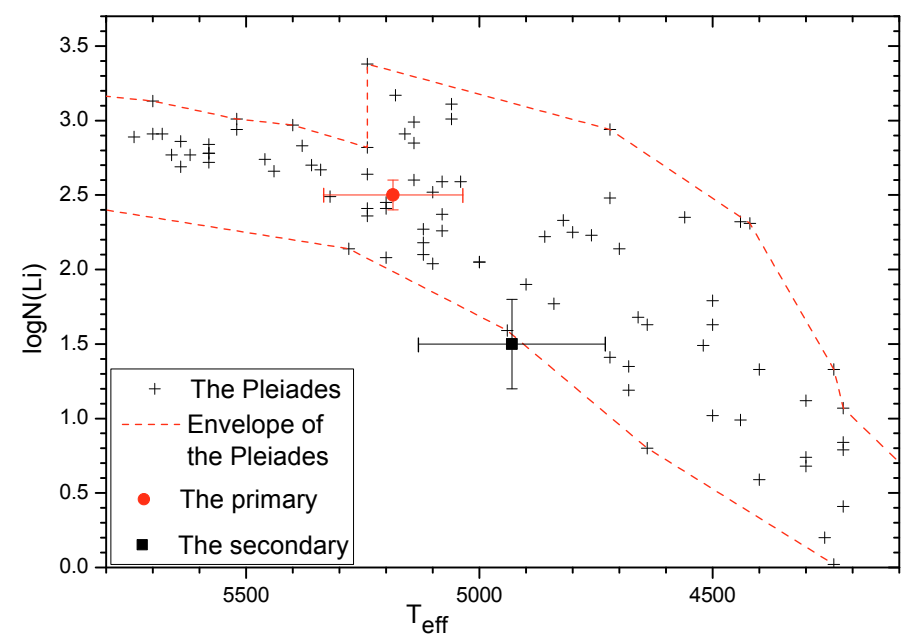

Fig. 6. Lithium abundance versus effective temperature for the individual components of HD 146875. The Pleiades ( 119 Myr) data and their envelopes are also plotted.

primary and $200 \mathrm{~K}$ for the secondary. The error in metallicity is 0.14 for the primary and 0.28 for the secondary. The gravity was determined by requiring that $\mathrm{Fe} \mathrm{I}$ and $\mathrm{Fe}$ II lines inferred the same iron abundance. The error in $\log g$ is 0.1 for the primary, and 0.2 for the secondary. The microturbulence was determined by requiring that there was a zero slope in the relation between $[\mathrm{Fe} / \mathrm{H}]$ and EW. The error in the microturbulence was about $0.3 \mathrm{~km} \mathrm{~s}^{-1}$ for the primary and $0.6 \mathrm{~km} \mathrm{~s}^{-1}$ for the secondary. Based on $T_{\text {eff }}$ and $\log g$, we calculated the bolometric magnitudes, $M_{\mathrm{bol}}$, the absolute visual magnitudes, $M_{\mathrm{V}}$, the masses, $M$, the radii, $R$, the orbital inclination, $i$, the spectroscopic distance, $D$, and the spectral types, $S p T$, of the components. We also measured the projected rotation velocities, $V \sin i$, of the components. All of the stellar atmospheric parameters and the derived quantities are listed in Table 2.

\subsection{Age determination}

We used two methods to investigate the age of HD 146875. The presence of a strong Li I absorption line is regarded as the primary indicator of stellar youth, so our first method was to compare the lithium abundance of components with those of the zero-age main-sequence (ZAMS) stars of the Pleiades (Soderblom 1993). The EW and the abundance of Li I line of the components are listed in Table 2. In Fig. 6, both of the components have similar lithium abundance to stars of the same $T_{\text {eff }}$ in the Pleiades, therefore we suggest that the binary has an age similar to that of the Pleiades, 119 Myr (Martin et al. 2001).

The second method was to compare the position of the components on the Hertzsprung-Russell diagram to the theoretical PMS evolutionary tracks of Siess (2000). We chose tracks computed for stars in the mass region 0.6 to $1.2 M_{\odot}$ with solar composition $(Z=0.02)$, including a moderate overshooting. In Fig. 7, both the positions of the components are close to the isochrone of $10^{8}$ years. We note that the primary detection appears to be more reliable than that of the secondary based on their spectral qualities.

The ages derived from these two methods are consistent with each other, and we propose that HD 146875 is a ZAMS binary with an ago of between 100 and $150 \mathrm{Myr}$. 


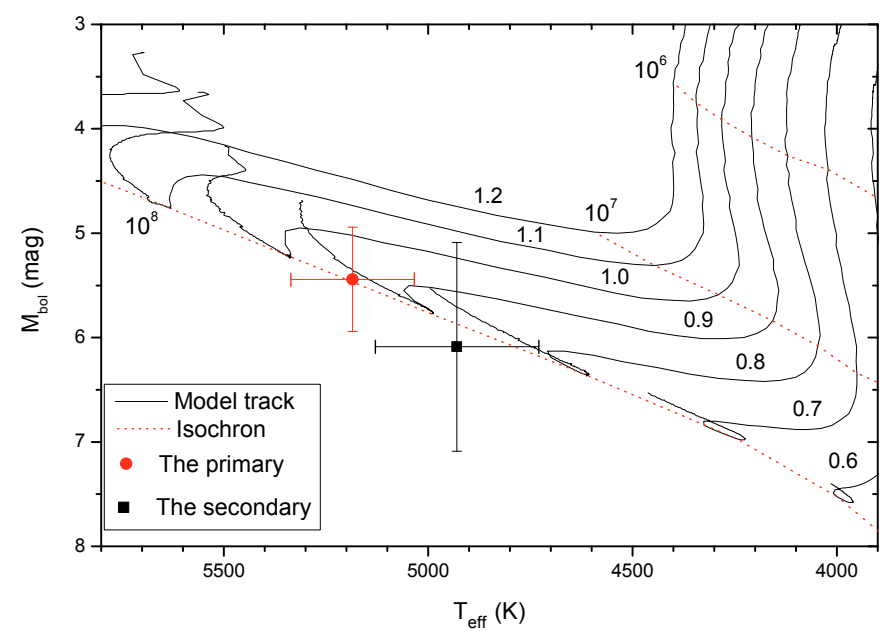

Fig. 7. Hertzsprung-Russell diagram for the components of HD 146875 , compared with the PMS evolutionary tracks of Siess. Isochrones are labeled in units of years.

\subsection{Orbital parameters derived from an RV curve fit}

To derive the RV of the centroid of the binary, we measured the RV of the components by cross-correlating their observed spectra with the spectrum of the RV standard star in IRAF. We adopt the RVs of the 3 standard stars, i.e., $\operatorname{RV}(\alpha$ Boo $)=-5.14 \pm$ $0.04 \mathrm{~km} \mathrm{~s}^{-1}, \operatorname{RV}(\beta \mathrm{Gem})=3.26 \pm 0.07 \mathrm{~km} \mathrm{~s}^{-1}$ and $\operatorname{RV}(\beta$ Oph $)=$ $-12.29 \pm 0.04 \mathrm{~km} \mathrm{~s}^{-1}$ (Stefanik et al. 1999). In this way, the typical precision of our RV measurement is $0.8 \mathrm{~km} \mathrm{~s}^{-1}$ for the primary component and $3.2 \mathrm{~km} \mathrm{~s}^{-1}$ for the secondary. All RVs of the components are presented in Table 1 . The RVs are plotted in Fig. 8 and used to complete an RV curve fit when the correlation function could be described by two different Gaussian profiles.

We adopted a circular orbit for the components based on the KOREL analysis result, and fitted the RV versus heliocentric Julian date (HJD) curve of each component with a function $y=\gamma+K * \sin \left(2 \pi \frac{x-x_{\mathrm{c}}}{P}\right)$, where $y$ is the RV of a component, $\gamma$ is the RV of the centroid of the binary, $\mathrm{K}$ is the amplitude of the $\mathrm{RV}$ curve, $x$ is HJD, $x_{\mathrm{c}}$ is the original phase of a curve in units of days, and $\mathrm{P}$ is the orbital period of the binary. The square of the correlation coefficient is 0.999 and 0.995 for the fit of the primary component and secondary, respectively, based on a least squares fit, which implies that these fits are reliable. All the results of the fit are presented in Table 3.

We note that the period derived in this way is 0.0021 days shorter than that derived with KOREL. We suggest that the KOREL result is more accurate because of its more robust disentangling of the components throughout the entire phase.

\subsection{The chromospheric activity}

The Ca II IRT lines (8498 ̊, $8542 \AA$, and $8662 \AA$ ) are good indicators of the optical chromospheric activity of a star. In all the observed spectra of HD 146875, clear filled-in emission is noticed in the Ca II IRT absorption lines (see Figs. 9 and 5). To separate the emission from the absorption lines, we adopted a spectral subtraction technique (Barden 1985). We chose the "nonactive" star 27 Ori (spectral type: G9 III-IV) as the comparison star for the primary component of HD 146875 and $\beta$ Gem (spectral type: K0 III) for the secondary, and compiled a synthetic "nonactive" composite model consisting of the spectra of the comparison stars. we then obtained the subtracted spectra by subtracting the composite model from the spectra of HD 146875

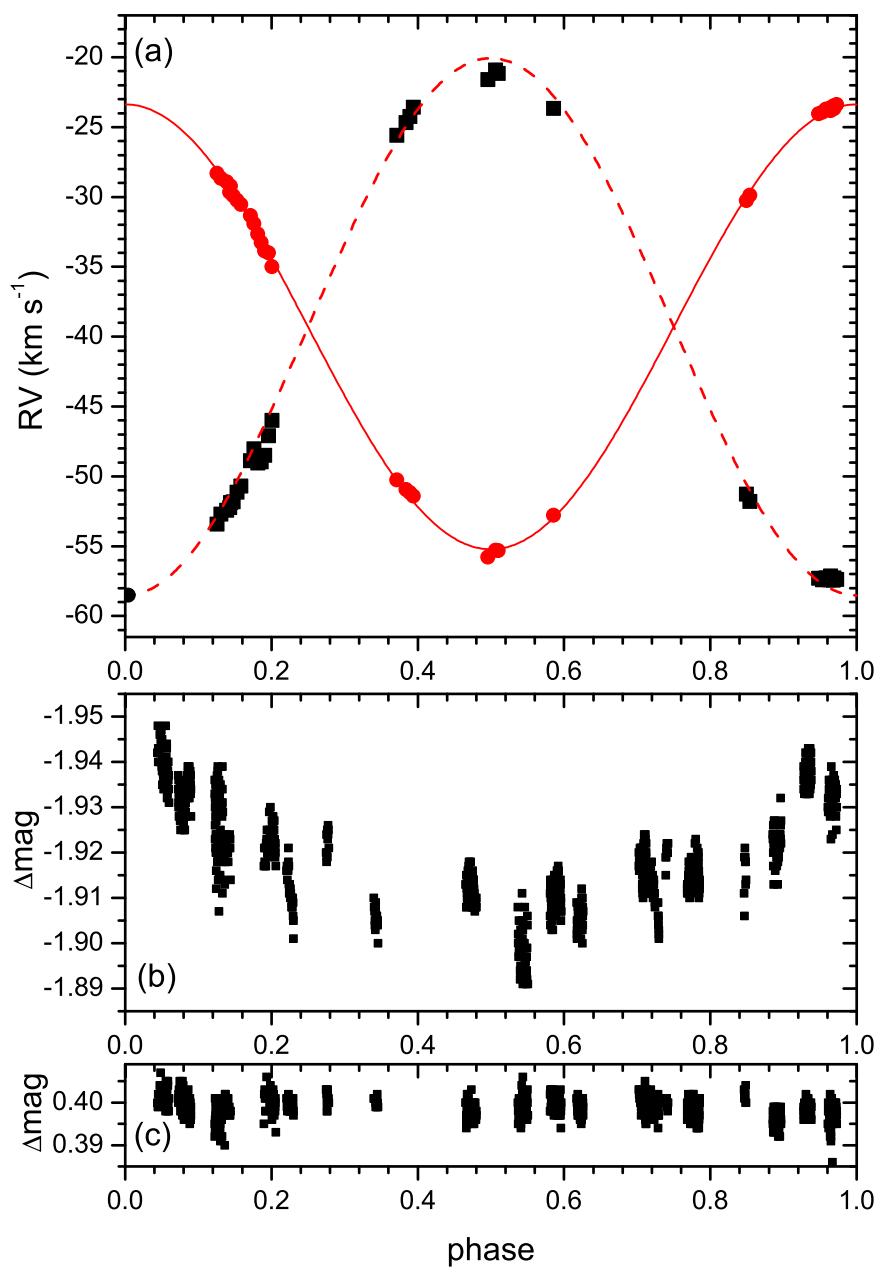

Fig. 8. The radial velocity curve for HD146875 in a), the contemporaneous V light curves of HD 146875 in b), and the contemporaneous $\mathrm{V}$ light curves of the comparison stars in $\mathbf{c})$. In a), the red points and black squares represent the RVs of the primary and secondary, respectively. The solid and dashed lines represent the circular solution for the primary and secondary.

Table 3. Results of RV curve fit and calculation.

\begin{tabular}{cc}
\hline \hline Parameter & Value \\
\hline$P^{a}$ (days) & $8.6209 \pm 0.0003$ \\
$P^{b}($ days $)$ & 8.62 \\
$\gamma\left(\mathrm{km} \mathrm{s}^{-1}\right)$ & $-39.29 \pm 0.06$ \\
$K_{\mathrm{P}}\left(\mathrm{km} \mathrm{s}^{-1}\right)$ & $15.9 \pm 0.8$ \\
$K_{\mathrm{S}}\left(\mathrm{km} \mathrm{s}^{-1}\right)$ & $19.2 \pm 3.2$ \\
$q$ & $0.83 \pm 0.08$ \\
\hline
\end{tabular}

${ }^{a}$ Obtained from RV curve fit.

${ }^{b}$ Obtained from photometric data.

(see Fig. 9). We measured the RV of the emission lines in the subtracted spectra, and found the RV of the emission line to be consistent with that of the primary with an error less than $0.7 \mathrm{~km} \mathrm{~s}^{-1}$ during the entire phase. This implies that the emission must then originate in the primary. We found no obvious emission from the secondary, which implies that the primary is more active than the secondary.

We calculated the ratio of excess emission $E W$, i.e., $\frac{E W_{8542}}{E W_{8498}}$, which is an indicator of the type of chromospheric structure producing the observed emission (Galvez et al. 2002). The values of the $\frac{E W_{8542}}{E W_{8498}}$ ratio are measured to be between 1.5 and 3 in solar plages, while the values are about 9 in solar prominences, 

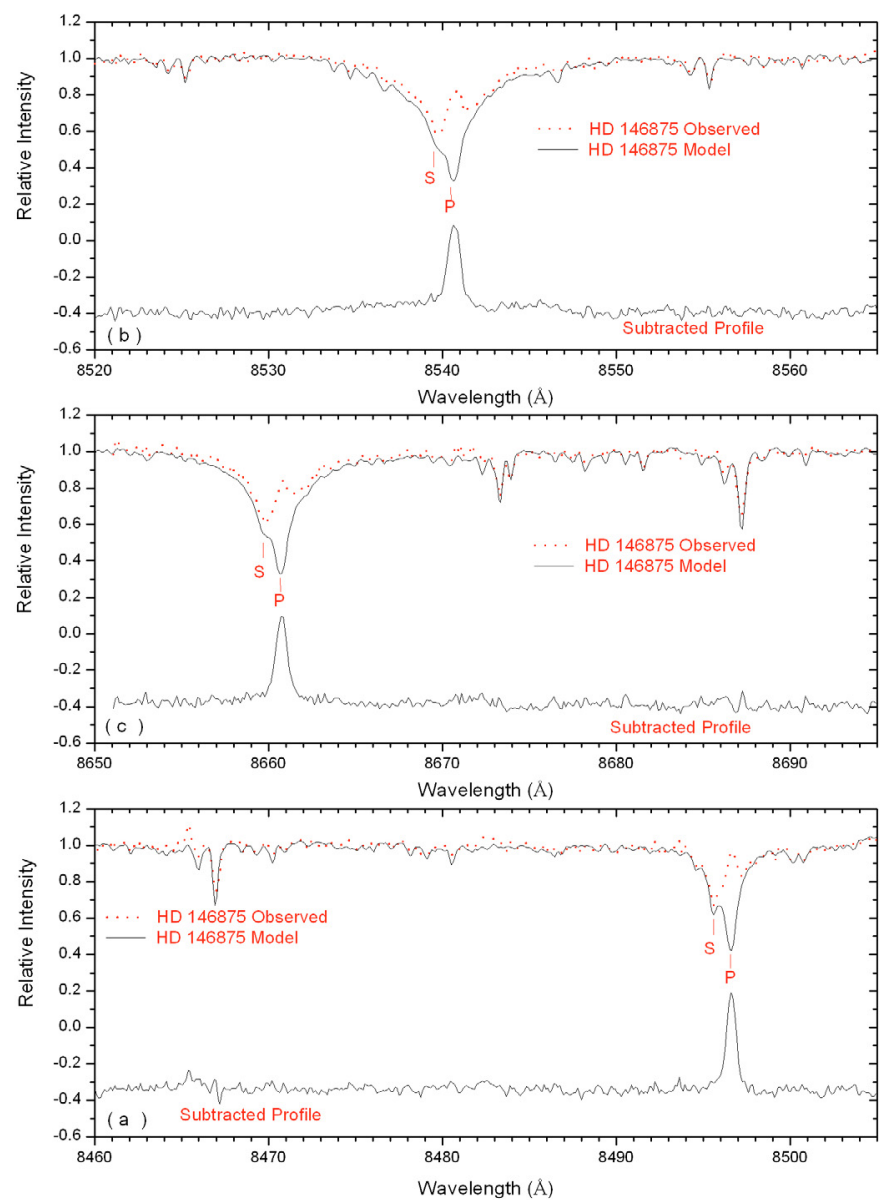

Fig. 9. a)-c) Are the sample spectrum, synthetic model, and subtracted emission profile in the regions of Ca II IRT $8498 \AA 8542 \AA$ and $8662 \AA$ lines respectively. The dotted line is an observed spectrum for HD 146875 taken at Xinglong Observatory with the TEK CCD at orbital phase 0.35 . The solid-line spectrum plotted onto the one observed is the synthetic "nonactive" composite model consisting of comparison stars similar in spectral type to the stellar components of the observed system. Shown at the bottom is the subtracted emission profile. The primary and the secondary components of the Ca II IRT profiles are indicated by $\mathrm{P}$ and $\mathrm{S}$ respectively.

the limit of an optically thin emitting plasma (Chester 1991). Because of the low values of the $\frac{E W_{8542}}{E W_{8498}}$ ratio that we found for the primary component in all of our spectra (ranging from 1.1 to 2.0), we suggest that the Ca II IRT emission of the primary star originates in plage-like regions. This phenomenon has also been detected in some other chromospherically active binaries, such as BK Psc (Galvez et al. 2002).

\subsection{Photometric variability}

Although the limited time range of our observations (2 months) does not enable a reliable period search to be made, the closest PDM fit was obtained for the synchronous period, 8.62 days. Based on a period of 8.6209 days, we plot the photometric data in Fig. 8.

These photometric data are of sufficiently good quality for us to determine whether the photometric variation is caused by ellipsoidal variations in the binary when studied together with our spectroscopic data (see Fig. 8). Firstly, the "one-peak-like" character of the light curve during the entire phase helps us realize that the variation in the light is caused by spots on the components rather than ellipsoidal variations. Secondly, We noticed that the period of the spots (which is also an indicator of the rotational period of the component related to these spots) provides an approximation of that of the orbit, which implies that HD 146875 is a synchronous binary system. Finally, we note that the brightest point of the light curve corresponds to the maximum RV of the primary, and the faintest point corresponds to the minimum RV of the primary, which implies that the spots are facing us when the primary is moving towards us at maximum speed (corresponding to minimum RV). Furthermore, the spots are most probably located on the primary component, because plage-like regions often appear close to spots.

\section{Conclusions}

Our conclusions can be summarized as follows. Firstly, HD 146875 is identified to be a ZAMS active double-lined SB for the first time on the basis of our 45 high-resolution spectra obtained between 2005 and 2008 at Xinglong Observatory, NAOC. Secondly, reliable orbital parameters were derived for HD 146875 by spectroscopic analysis, and the stellar atmospheric parameters of the components were derived from an abundance analysis (based on the list in Table 2). We have derived some fundamental parameters of the binary, such as the absolute bolometric magnitudes, masses, radii, orbital inclination, and spectroscopic distance (list in Table 2). We have found that the primary is the more active component of the binary based on the analysis of Ca II IRT emissions. By considering the photometric observations performed in 2008, we have also found no obvious ellipsoidal distortion but a variation of about $0.057 \mathrm{mag}$ caused by large spots most probably on the primary component of the binary. Furthermore, the RV curve and the light curve imply that HD 146875 is already a synchronous system and that the spots face us when the primary moves towards us at its maximum speed.

Acknowledgements. We would like to thank the anonymous referee for very helpful comments and suggestions. We acknowledge Prof. Hadrava for providing the KOREL routine. We acknowledge Prof. Jingyao Hu for the revealing suggest in our work and we always have a better understanding of the issue after each discussion with him. We thank Dr. Jing Wang, Dr. Caina Hao, my classmates Wei Su, Fengchun Zhang, Xuhui Han, and Weikang Zheng for their warm-hearted helps in our data reduction. We thank Liping Xin, Huali Li, Wenxiu Jiang, Qichen Feng, and Qin Lin for their sincere helps in photometric observations. This work was supported by the National Natural Science Foundation of China through grants Nos. 10473013 and 10503006. This work was also supported by the National Basic Research Program of China through grant No. 2009CB824800. This research has made use of the SIMBAD database, operated at CDS, Strasbourg, France.

\section{References}

Barden, S. C. 1985 , ApJ, 295, 162

Cargile, P. A., Stassun, K. G., \& Mathieu, R. D. 2008, ApJ, 674, 329

Chen, Y. Q., Nissen, P. E., Zhao, G., Zhang, H. W., \& Benoni, T. 2000, A\&AS, 141,491

Chester, M. M. 1991, Ph.D. Thesis, Pennsylvania State Univ.

Covino, E., Melo, C., Alcalá, J. M., et al. 2001, A\&A, 375, 130

Galvez, M. C., Montes, D., Fernández-Figueroa, M. J., et al. 2002, A\&A, 389, 524

Hadrava, P. 1995, A\&AS, 114, 393

Martin, E. L., Dahm, S., \& Pavlenko, Y. 2001, ASP Conf. Ser., 245, 349

Prato, L., Montes, D., Fernández-Figueroa, M. J., et al. 2002, ApJ, 569, 863

Ryan S. G. 1998, A\&A, 331, 1051

Schwope, A. D., Hasinger, G., Lehmann, I., et al. 2000, AN, 321, 1

Siess, L., Dufour, E., \& Forestini, M. 2000, A\&A, 358, 593

Soderblom D. R., Jones, B. F. Balachandran, S., et al. 1993, AJ, 106, 1059

Stefanik, R. P., Latham, D. W., \& Torres, G., et al. 1999, ASPC, 185, 354

Stellingwerf, R. F. 1978, ApJ, 224, 953

Zhao, G., \& Li, H.-B. 2001, Chinese J. Astron. Astrophys., 1, 555

Zhao, J. K., Zhao, G., \& Chen, Y. Q. 2007, PNAOC, 4, 153 